\title{
Effect of propranolol and phentolamine on myocardial necrosis after subarachnoid haemorrhage
}

\author{
G NEIL-DWYER，P WALTER，J M CRUICKSHANK， B DOSHI，P O'GORMAN
}

British Medical fournal, 1978, 2, 990-992

\section{Summary and conclusions}

A study was set up to assess the effect on the clinical course of subarachnoid haemorrhage (SAH) of giving propranolol $80 \mathrm{mg}$ eight-hourly plus phentolamine $20 \mathrm{mg}$ three-hourly by mouth for three weeks. Out of the 90 patients studied, 14 died. Two of the deaths occurred in an open pilot study of 10 patients, the remaining 12 deaths occurring in patients in a randomised doubleblind placebo-controlled study. Postmortem examination was carried out on 12 of the patients, six of whom had been receiving placebo and six propranolol plus phentolamine. Necrotic myocardial lesions were present in the hearts of all six patients (age range 30-59 years) who died while taking placebo (all had had abnormal electrocardiograms (ECGs)). In contrast, no necrotic lesions were found in the hearts of the six patients (age range 28-59) who died while receiving the drugs (all had previously had normal ECGs).

We conclude that the necrotic myocardial lesions were induced by catecholamines and that propranolol had a cardioprotective effect. While death from a further haemorrhage in cases of SAH is not affected by propranolol and phentolamine, propranolol may have a beneficial effect in other potentially lethal stresses.

\section{Introduction}

The bizarre electrocardiographic abnormalities that are often associated with subarachnoid haemorrhage (SAH) probably result from increased sympathetic outflow. ${ }^{2}$ Propranolol abolishes many of these changes. ${ }^{3}$ Simulated SAH in an animal model is accompanied by similar electrocardiographic changes and subendocardial necrotic lesions; both abnormalities may be prevented by surgical (C2 cordotomy) or medical (propranolol) intervention." The necrotic myocardial lesions are identical with those induced by administration of catecholamines ${ }^{5}$ and those found in the hearts of patients who have died from SAH. ${ }^{6}$ These cardiac lesions may well influence the morbidity and mortality of such conditions as SAH and head injury.?

The present study was set up to investigate the effect of propranolol on the clinical course of SAH and in particular on the incidence of myocardial necrotic lesions. Phentolamine, an alpha-blocker, was also given so that the effect of both drugs on the lethal course of cerebral arterial spasm after a SAH might be studied.

Brook General Hospital, Woolwich, London SE18 4LW

G NEIL-DWYER, MS, FRCS, consultant neurosurgeon

$P$ WALTER, BSC, FRCS, neurosurgical registrar

B DOSHI, MRCPATH, consultant neuropathologist

P O'GORMAN, MRCPATH, consultant, chemical pathology department

Pharmaceuticals Division, ICI Limited, Alderley Park, Macclesfield, Cheshire SK10 4TG

J M CRUICKSHANK, DM, MRCP, medical adviser

\section{Patients and methods}

We studied 90 consecutive patients with SAH proved by lumbar puncture who were admitted to the South-east Thames regional neurosurgical unit at the Brook General Hospital between November 1976 and April 1978. Only patients who were admitted into the unit within 48 hours after their haemorrhage were placed in the trial. Those aged over 60 or with a history of hypertension, ischaemic heart disease, congestive cardiac failure, or obstructive airways disease were omitted.

The first 10 patients entered a pilot study set up to guard against an unexpected interaction between patient and treatment. All 10 were given $20 \mathrm{mg}$ of phentolamine three-hourly plus $80 \mathrm{mg}$ of propranolol eight-hourly for three weeks. When necessary the tablets were crushed and given by nasogastric tube. The next 80 patients entered the randomised, double-blind between-patient study; 40 received the above treatment for three weeks and 40 received matching placebo tablets. The patients were randomised in pairs by computer.

Clinical assessments of level of consciousness, neurological state, mean pulse rate, and blood pressure were recorded daily. On days $1,3,7,10,14$, and 21 a standard 12-lead electrocardiogram (ECG) was obtained (reported blind by JMC) and a 24-hour urine collection started to measure the excretion rates of urinary catecholaminesnamely, adrenaline, noradrenaline, 3-methoxy-4-hydroxymandelic acid, and metadrenaline. The methods used to measure these excretion rates have been described elsewhere. ${ }^{8-10}$ Cerebral angiography was performed when considered to be clinically necessary. The anaesthetists broke the code for individual patients at operation, but this information was not available to the rest of the staff. Drug treatment continued during the preoperative and postoperative periods.

Postmortem examination was carried out on all patients who died for whom permission was obtained, particular attention being paid to the histology of the hypothalamus and heart. Three blocks were taken from each hypothalamus at the levels of the optic chiasm, the tuber cinereum, and the mammillary bodies for paraffin section. The sections were stained with haematoxylin and eosin, haematoxylin and van Gieson, and Luxol fast blue and cresyl violet. The coronary arteries were examined in all cases, and one block was taken from each of the five following areas of the left ventricle for paraffin section: posterior wall, lateral wall, anterior wall, interventricular septum, and apex. The sections were stained with haematoxylin and eosin and haematoxylin and van Gieson. The pathologist routinely examines the hypothalamus and heart of every patient who dies after a SAH and so was unaware of which patients were in the trial.

\section{Results}

Fourteen patients died, of whom two were in the open pilot study and 12 in the blind control study. Of the latter 12 deaths, six occurred in patients taking placebo and six in patients taking propranolol and phentolamine. Postmortem examination was not carried out in two cases, and so this analysis is confined to the 12 patients remaining (six of whom received placebo and six the drugs).

Of the 12 patients who died, the two groups were reasonably well matched (table I) except for the preponderence of men among the patients receiving the drugs and of women among those receiving placebo. Modes of death differed between the two groups. In the treated group all six deaths were due to further haemorrhage, while in the untreated group two patients died from haemorrhage and the remaining four died after a gradual deterioration due to cerebral arterial spasm, though one of these patients may have had a pulmonary embolus. Two of these four died after surgery. The incidence of radiographic evidence of cerebral arterial spasm was similar in both groups.

Table II shows the haemodynamic, metabolic, and pathological changes in the two groups. The slower mean pulse rate in the treated group $(65.0$ compared with 80.9 beats $/ \mathrm{min}, \mathrm{P}<0.05)$ is evidence of 
TABLE I-Data on 12 patients on whom necropsy was carried out

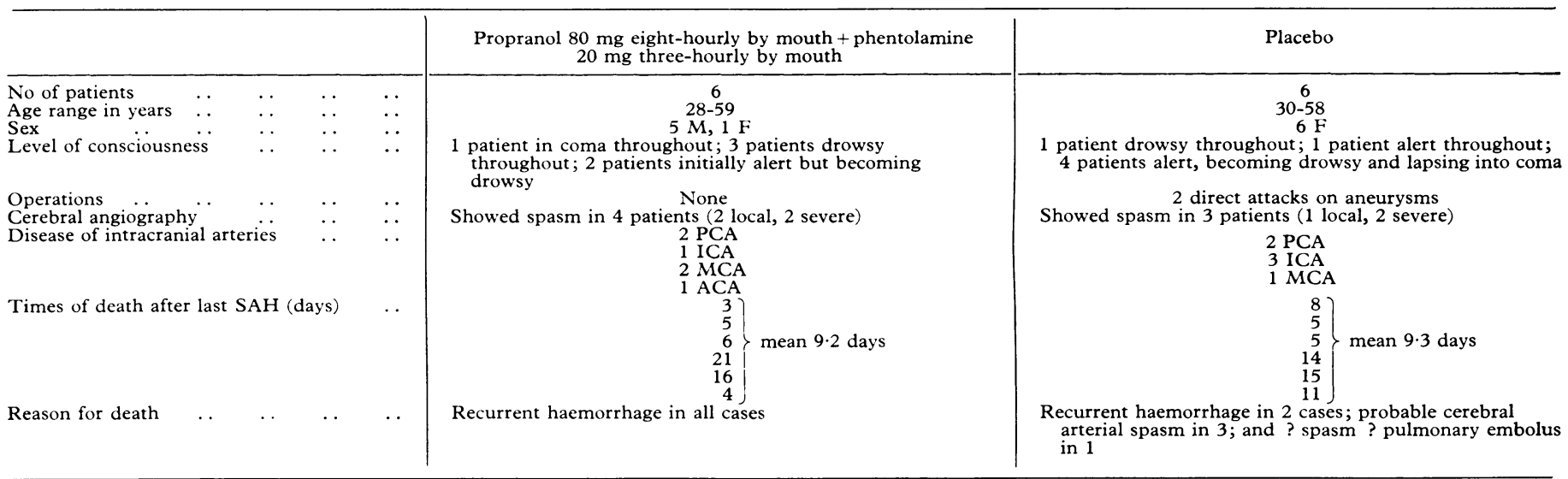

$\mathrm{PCA}=$ Posterior communicating artery aneurysm. $\mathrm{ICA}=$ Internal carotid artery aneurysm. $\mathrm{MCA}=$ Middle cerebral artery aneurysm. $\mathrm{ACA}=\mathrm{Anterior}$ communicating artery aneurysm.

TABLE II-Clinical details and findings at necropsy in 12 patients who died

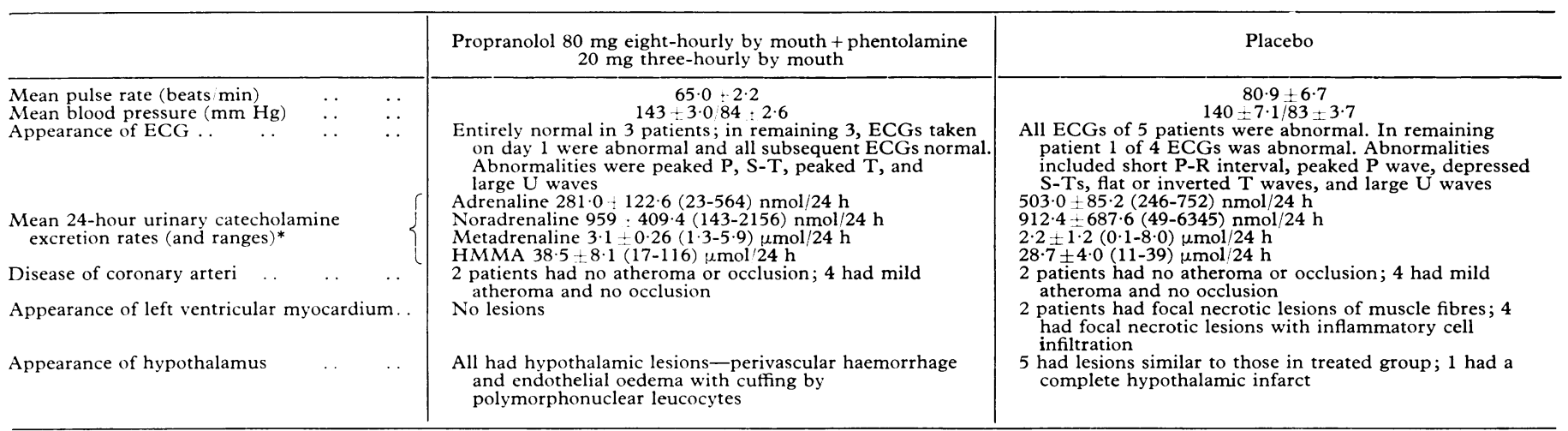

HMMA = 3-Methoxy-4-hydroxymandelic acid.

*Normal ranges: adrenaline 0-275 nmol $/ 24 \mathrm{~h}$; noradrenaline $110-1100 \mathrm{nmol} / 24 \mathrm{~h}$; metadrenaline $0-4 \cdot 0 \mu \mathrm{mol} / 24 \mathrm{~h} ; \mathrm{HMMA} 10-35 \mu \mathrm{mol} / 24 \mathrm{~h}$. Conversion: SI to traditional units -Adrenaline: $1 \mathrm{nmol} / 24 \mathrm{~h} \approx 0 \cdot 18 \mu \mathrm{g} / 24 \mathrm{~h}$. Noradrenaline: $1 \mathrm{nmol} / 24 \mathrm{~h} \approx 0 \cdot 17 \mu \mathrm{g} / 24 \mathrm{~h}$. Metadrenaline: $1 \mu \mathrm{mol} / 24 \mathrm{~h} \approx 0 \cdot 18 \mathrm{mg} / 24 \mathrm{~h}$. $\mathrm{HMMA}$ :
$1 \mu \mathrm{mol} / 24 \mathrm{~h} \approx 0 \cdot 2 \mathrm{mg} 24 \mathrm{~h}$.

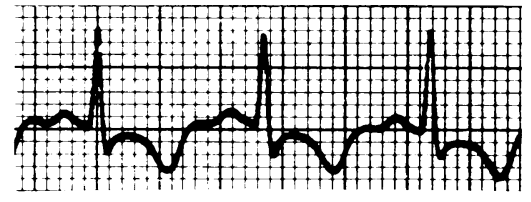

FIG 1-Appearance of ECG (lead V5) in patient taking placebo, showing $\mathrm{S}-\mathrm{T}$ depression and $\mathrm{T}$-wave inversion.

beta-blockade. There was also a pronounced difference in electrocardiographic pattern between the two groups, the basic normality of the ECGs of the treated group contrasting with the abnormality of all those in the placebo group. Figure 1 shows the ECG of one of the patients in the placebo group. The "stressed" condition of the patients was evidenced by the fairly high mean concentrations of urinary catecholamines in both groups. Although there was no statistical difference between the two groups the mean urinary adrenaline concentration was high in the untreated group and virtually normal in the treated group. Several patients at times had urinary catecholamine concentrations equivalent to those found in patients with phaeochromocytomas. No severe coronary arterial disease was seen in any patient.

None of the six patients in the group receiving the drugs had necrotic myocardial lesions. In contrast, all six patients who died while taking placebo had focal necrotic lesions of the left ventricular muscle fibres with or without inflammatory cell infiltration. Figure 2 shows an example of such a lesion (which arose in the patient whose ECG is shown in fig 1). All patients in both groups had hypothalamic lesions comprising perivascular haemorrhages with oedema of the vessel wall affecting the endothelial cells and perivascular cuffing of polymorphonuclear leucocytes. One patient in the untreated group had a complete infarct of the hypothalamus.

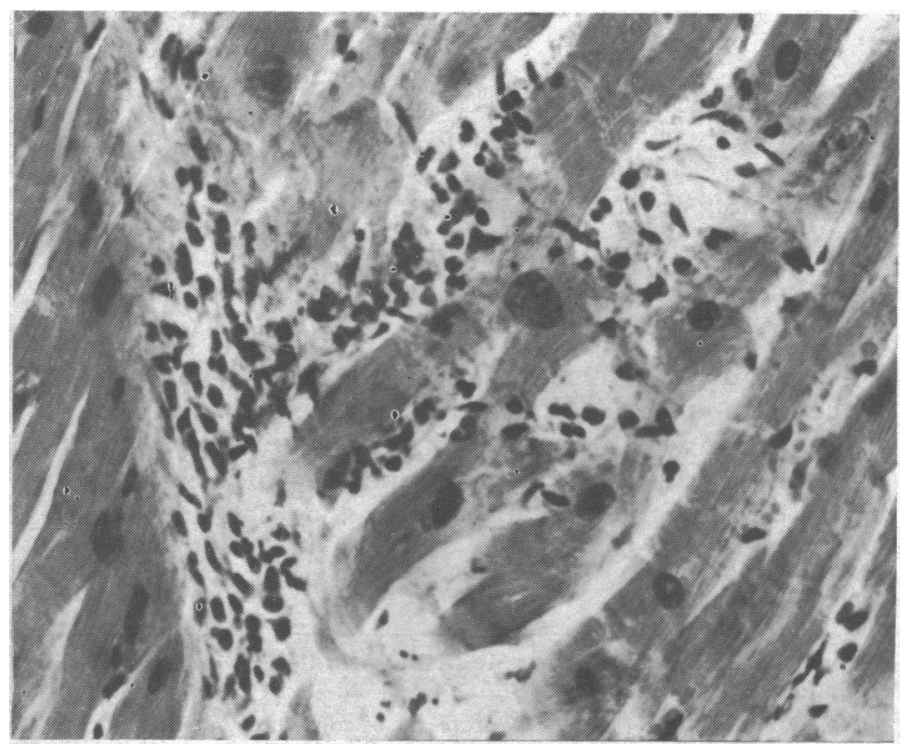

FIG 2-Necrotic muscle fibre in heart with interstitial inflammatory cell infiltration. (Haematoxylin and eosin. $\times 160$ ) 


\section{Discussion}

Simulated SAH, ${ }^{4}$ hypothalamic stimulation, ${ }^{11}$ and parenteral administration of catecholamines ${ }^{5}$ in animals produce necrotic myocardial lesions, which may be prevented by severing the cord at $\mathrm{C} 2$ level or by administering reserpine or propranolol. ${ }^{4}$ A possible effect of the hypothalamic lesions after a $\mathrm{SAH}^{12}$ is to produce an abnormal reaction of the autonomic nervous system resulting in persistantly high excretion rates of urinary catecholamines. ${ }^{2}$ The close association between the hypothalamus and the autonomic nervous system, coupled with the high correlation between hypothalamic and myocardial lesions after $\mathrm{SAH}^{13}$ points to a catecholamine-linked origin of the myocardial necrosis.

The pathological results of this study should be interpreted in the light of results (unpublished) of a recent survey, which showed that out of 54 patients who died of SAH at the Southeast Thames regional neurosurgical unit, 49 had hypothalamic lesions similar to those found in this study and 42 of these patients (78\% of the whole) had necrotic myocardial lesions. Thus the fact that in our study all six patients who died in the drug-treated group had "clean hearts" (with normal ECGs) while all six in the placebo-treated group had focal necrotic myocardial lesions (and abnormal ECGs) becomes important. Due to the probable absence of alpha-receptors in the myocardium $^{14}$ we presume that propranolol rather than phentolamine was the agent responsible.

What are the clinical implications of these results ? Excessive amounts of catecholamines may induce heart failure, as in the cardiomyopathy associated with phaeochromocytomas. ${ }^{15}$ Some other types of congestive cardiomyopathy may well result from an abnormal interaction between catecholamines and the myocardium and benefit from beta-blockade. ${ }^{16} \mathrm{~A}$ recent report ${ }^{17}$ described such a cardiomyopathy, which was cured by stopping treatment with ephedrine. Some authors consider that catecholamines play a crucial part in the onset of myocardial infarction, ${ }^{18}$ thus suggesting an explanation for the protective effect of beta-blockade on the ischaemic myocardium. Despite its "cardioprotective effect," propranolol did not influence mortality in cases of SAH and accompanying myocardial damage. This is because lethal secondary events such as a second SAH are not critically influenced by propranolol or phentolamine, though the results of this preliminary study suggest a possible protective action of these two drugs with regard to cerebrovascular spasm. In other "stress areas" with associated myocardial necrotic lesions and unstable cardiac rhythms, such as head injuries, ${ }^{7}$ beta-blockade may have an important role. The source of hearts for cardiac transplantation is another important, though perhaps more remote, factor to be considered. If, as is often the case, the donor has died after a cerebrovascular accident or head injury then early administration of a beta-blocker to the potential donor might be wise.

We would like to thank Dr D M Burley of Ciba Laboratories for supplying the phentolamine for the clinical trial.

Requests for reprints should be addressed to G Neil-Dwyer.

\section{References}

1 Tomomatsu, T, et al, Fapanese Circulation fournal, 1964, 28, 905.

2 Neil-Dwyer, G, et al, fournal of the Neurological Sciences, 1974, 22, 375.

${ }^{3}$ Cruickshank, J M, Neil-Dwyer, G, and Lane, J, Cardiovascular Research, 1975, 9, 236.

4 Greenhoot, J H, and Reichenbach, D D, fournal of Neurosurgery, 1969 30, 521.

5 Reichenbach, D D, and Benditt, E P, Human Pathology, 1970, 1, 125.

6 Conor, R C R, American Heart fournal, 1969, 78, 145.

7 Hersch, C, Circulation, 1961, 23, 853.

${ }^{8}$ Sobel, C, and Henry, R J, American Fournal of Clinical Pathology, 1957, 27, 240.

- Pisano, J J, Clinica Climica Acta, 1960, 5, 406.

10 Pisano, J J, Crout, J R, and Abraham, D, Clinica Climica Acta, 1962, 7, 285.

11 Melville, K I, et al, American fournal of Cardiology, 1963, 12, 781.

12 Crompton, M R, Brain, 1963, 86, 301.

13 Doshi, R, and Neil-Dwyer, G, Fournal of Neurology, Neurosurgery and Psychiatry, 1977, 40, 821.

14 Waagstein, F, et al, British Heart fournal, 1975, 37, 1022.

15 Van Vliet, P D, Burchell, H B, and Titus, J L, New England fournal of Medicine, 1966, 274, 1102.

16 Goodman, L S, and Gilman, A, The Pharmacological Basis of Therapeutics, 4th edn, p 406. New York, Macmillan, 1971.

17 Van Mieghem, W, Stevens, E, and Cosemans, J, British Medical fournal, $1978,1,816$.

18 Waldenstrom, A P, Hjalmarson, A C, and Thornell, L, American Heart fournal, 1978, 95, 43.

(Accepted 2 August 1978)

\title{
National Childhood Encephalopathy Study : an interim report
}

\author{
DAVID L MILLER, EUAN M ROSS
}

British Medical fournal, 1978, 2, 992-993

\section{Summary and conclusions}

Data from the first year of the National Childhood Encephalopathy Study were reviewed to see whether any relation was apparent between pertussis vaccination and brain disease. Three hundred and eighty-seven cases of encephalitis and other specified neurological

\footnotetext{
Department of Community Medicine, Middlesex Hospital Medical School, London NW10 7NS

DAVID L MILLER, MD, FRCP, professor of community medicine

EUAN M ROSS, MD, MRCP, senior lecturer in child health and community medicine
}

conditions in which the children were admitted to hospital were reported, of which 267 satisfied the study criteria. Control children were matched for age with the index cases, and medical and immunisation histories were reviewed. Few of the index cases had been vaccinated within 28 days before admission to hospital, so that no close association between vaccination and brain disease existed in most cases.

The number of children who had recently been immunised was too small for any statistically useful conclusion to be reached about the risk associated with pertussis vaccine. The study is continuing.

\section{Introduction}

Allegations that immunisation against pertussis carries a risk of causing permanent brain damage in an appreciable 